\title{
4 Überblick: Die deutsch-italienische Besetzung Südosteuropas
}

Obwohl Südosteuropa im Zweiten Weltkrieg gemeinhin als „Nebenkriegsschauplatz" galt, waren in der Region doch bis zu 800.000 deutsche Soldaten gebunden. ${ }^{1}$ Dies obgleich der Balkan und Griechenland nie Teil von Hitlers Großmachtplänen gewesen waren, ${ }^{2}$ und er seinem Verbündeten Mussolini diese Einflusssphäre gegönnt hätte. ${ }^{3}$ Dieser war nach jahrelanger Planung und schließlich erdrückender Einflußnahme auf den albanischen König Ahmed Zogu bereits am 7. April 1939 in Albanien einmarschiert, mit dem Ziel, eine italienische Kolonie zu etablieren und den jungen Staat als Teil des „mare nostro“ in das faschistische Imperium zu integrieren. ${ }^{4}$

Am 1. September desselben Jahres brach mit dem deutschen Angriff auf Polen der Zweite Weltkrieg aus. Obwohl Italiens Griechenlandfeldzug im Herbst/ Winter 1940/41 scheiterte, sah die Situation für Hitler zunächst entspannt aus: Seine südwestliche Flanke würde beim geplanten Angriff auf die Sowjetunion durch Vasallenstaaten gedeckt sein. Dies sollte sich Ende März 1941 ändern. Nachdem am 25. März 1941 die jugoslawische Regierung in Belgrad den Dreimächtepakt der Achsenmächte - wie zuvor Rumänien (20. November 1940) und Bulgarien (1. März 1941) - unterzeichnet hatte, entschieden sich mehrere Generale zum

1 Vgl. http://www.zukunft-braucht-erinnerung.de/volksdeutsche-manipuliermasse-in-der-ssdivision-prinz-eugen/ (Stand: 25.8.2017). Vgl. zu Südosteuropa als „Nebenfront“ detailliert Klaus Schmider, Der jugoslawische Kriegsschauplatz (Januar 1943 bis Mai 1945), in: Karl-Heinz Frieser (Hg.), Die Ostfront 1943/44: Der Krieg im Osten und an den Nebenfronten, Das Deutsche Reich und der Zweite Weltkrieg, Bd. 8, München: Deutsche Verlags-Anstalt 2007, S. 1009-1088. 2 Vgl. Hans Umbreit, Die deutsche Herrschaft in den besetzten Gebieten, in: Kroener, Bernhard R. u. a.: Organisation und Mobilisierung des deutschen Machtbereichs, hg. vom Militärgeschichtlichen Forschungsamt Potsdam, Das Deutsche Reich und der Zweite Weltkrieg, Bd. 5/2, Stuttgart: Deutsche Verlags-Anstalt 1999, S. 71.

3 Vgl. Ciano, Tagebuch, 22.5.1939, S. 92.

4 Konkrete Hinweise auf eine Kolonie liefert etwa die Tagebuchnotiz Cianos zum Bau des Dorfes Pater bei Tirana, in welchem fünfhundert aus dem Ausland zurückgekehrte Italiener wohnen sollten. Ciano, Tagebuch, 13.6.1939, S. 104. Auch Pearson schreibt, dass bereits am 10. April 1939 die ersten 22.000 italienischen Siedler den Hafen von Bari Richtung Albanien verlassen hätten. Vgl. Pearson, Albania in Occupation and War, S. 4. Für die Eingliederung Albaniens in das faschistische Imperium spricht beispielsweise die Krönung Vittorio Emanueles III. zum König von Italien und Albanien in Personalunion. Gegenüber Lord Perth vertrat Ciano die Meinung, dieser Dynastiewechsel von König Zogu zu König Vittorio Emanuele III. sei ein innenpolitischer Vorgang und gehe daher niemanden etwas an. Vgl. Ciano, Tagebuch, 19.4.1939, S. 77.

Ә Open Access. ( 2021 Franziska Anna Zaugg, publiziert von De Gruyter. (c) BY Dieses Werk ist lizenziert unter einer Creative Commons Namensnennung 4.0 International Lizenz. https://doi.org/10.1515/9783110730777-004 
Putsch und zwangen die Regierung Dragiša Cvetković und Prinz Paul Karađorđević abzudanken. An seine Stelle trat der minderjährige König Peter II. Hitler hätte ein verbündetes Jugoslawien als Flankenschutz bevorzugt, wäre es doch kräfte- und zeitsparender gewesen. Unter diesen neuen Voraussetzungen sah er sich aber gezwungen, Jugoslawien zu besetzen. ${ }^{5}$

Am 6. April 1941 marschierte die Wehrmacht mit 33 Divisionen in Jugoslawien und Griechenland ein. Unterstützt wurden die deutschen Truppen durch 38 italienische Divisionen und weitere ungarische Verbände. Bereits nach wenigen Tagen kapitulierten die beiden Staaten: Jugoslawien am 17. und Griechenland am 23. April. Die deutsche Invasion hatte eine komplette Neuordnung der südosteuropäischen Gebiete zur Folge: Serbien, das Banat, das nördliche Kosovo sowie ein Teil des Sandžaks kamen unter deutsche Militärverwaltung, der größte Teil Kosovos sowie Grenzgebiete Montenegros und Mazedoniens wurden Teil „Großalbaniens“ unter italienischer Herrschaft. Der östlichste Teil Kosovos sowie der größte Teil Mazedoniens wurden Bulgarien einverleibt, das auch Ansprüche auf Teile Griechenlands und Jugoslawiens erhob. Montenegro und die kroatischen Küstengebiete wurden Teil des italienischen Imperiums. Im größten Teil Kroatiens aber, zusammen mit Bosnien und der Herzegowina, Slawonien und Syrmien, entstand der deutsche Vasallenstaat, NDH, unter der Führung von Ante Pavelić, der gleichzeitig auch der Ustaša-Bewegung vorstand. ${ }^{6}$ Slowenien wurde zwischen Italien, Deutschland und Ungarn aufgeteilt, dass ebenfalls jugoslawische Gebiete für sich reklamierte. Griechenland schließlich wurde zwischen Italien, Deutschland und Bulgarien aufgeteilt. ${ }^{7}$

Der Sieg über Königreich Jugoslawien konnte zwar binnen weniger Tage errungen werden, die langfristige Befriedung Südosteuropas gelang jedoch nicht. Wehrmacht und Waffen-SS kämpften fast vier Jahre gegen zuerst kleine Gruppen verschiedener Aufständischer und später gegen eine exponentiell anwachsende, gut trainierte, gut ausgerüstete und motivierte Partisanenarmee nach westeuropäischem Vorbild, welche als Guerrilla-Formationen auch topographisch

5 Vgl. Jozo Tomasević, War and Revolution in Yugoslavia, S. 47.

6 Ustaša - Hrvatska Revolucionarna Organizacija heisst soviel wie Rebell - Kroatische Revolutionäre Organisation und war eine rechtsextreme geheime paramilitärische Organisation, die 1929 von Ante Pavelić ins Leben gerufen wurde. Nach dem Balkanfeldzug und Pavelićs Einsetzung als Staatschef verfolgte die Ustaša vor allem Serben und Juden, aber auch Muslime. Korb geht von einer Zahl von ungefähr 500.000 ermordeten Personen zwischen 1941-1945 aus. Vgl. Alexander Korb, Im Schatten des Weltkriegs, S. 12. Vgl. zur Regierung von Satellitenstaaten auch Leleu, La Waffen-SS, S. $179 \mathrm{f}$.

7 Tomasević, War and Revolution in Yugoslavia, S. 61-64. 
im Vorteil waren. ${ }^{8}$ Es war keine einzelne „Nebenfront“, kein einzelner „Krieg“, vielmehr waren es zahlreiche parallel verlaufende, manchmal sich überschneidende, bürgerkriegsähnliche Konflikte. Es handelete sich, um die Worte Korbs wiederzugeben, um „komplexe Gewalträume“, in welchen es keine klaren Fronten gab und oft schwierig zu erkennen war, wer mit dem Gegner zusammenarbeitete und wer auf der eigenen Seite kämpfte. ${ }^{9}$ Im Januar 1945 erklärte Generalfeldmarschall Maximilian von Weichs, Oberbefehlshabers der Heeresgruppe F, die Eigenart dieses Kriegsschauplatzes in kolonialistischen Worten:

Seit Abschluss des Balkanfeldzuges gilt der Südosten als Nebenkriegschauplatz. Drei Jahre lang musste in diesem Raum die deutsche Herrschaft ohne ausreichende Kräfte und mit ungenügenden Hilfsmitteln gegen zunehmenden Feinddruck aufrechterhalten werden. Die Gräberfelder in allen größeren Städten des Südostens sprechen eine ernste Sprache und künden doch nichts von den Tausenden, die irgendwo in den unendlichen Berglanden oder auf dem Meer in treuer Pflichterfüllung einsam geendet sind. Dies alles geschah, obwohl kein Angriff von außen gegen die weitgespannten Küstenfronten erfolgt ist, obwohl es nur Banden gab, gegen die es zu kämpfen galt auf dem Balkan! Aber es war kein „Kleinkrieg“, nein, es war ein allesumfassender Aufruhr, ein Losbrechen jahrhundertealter Leidenschaften triebhafter Naturvölker, überlagert vom Zusammenstoß der heutigen Zeit beherrschenden polaren Weltanschauungen, ein Ringen ohne Anfang und ohne Ende, ein Kämpfen ohne Fronten, ein Fechten wider aller Regeln moderner Kriegskunst. ${ }^{10}$

Als die deutschen Truppen 1941 in Jugoslawien einmarschierten, wurden glimmende Konflikteherde neu entfacht und solche, die bereits flammten - oft durch Zutun der Besatzungsmächte - noch verstärkt. Auch Major Hermann K. Frank beschreibt, wie die Eindrücke von der lokalen Konfliktsituation und ihrer Unüberschaubarkeit im Partisanenkampf ihn und seine Soldaten überwältigten:

Sippen, Stämme, Religionsgemeinschaften und die vielen politischen Interessengruppen bekämpften sich gegenseitig und lieferten sich, oft aus geringem Anlass, heftige, verlustreiche Kämpfe. Dazu herrscht in Albanien auch heute noch das grausame Gesetz der Blutrache. Im ganzen Land tobte wilder Kleinkrieg, als die deutschen Einheiten ins Land kamen. Überall, auch in den Städten, wurde scharf geschossen. Dabei wusste von den Deutschen keiner, wer überhaupt auf wen schoss und warum geschossen wurde. Die politische Lage war sehr unklar; die Verhältnisse verworren. ${ }^{11}$

8 Vgl. Schmidhuber, „Zusammenfassender Bericht“, 2.10.1944, BArchF, RS 3-21/1, S. 5. Klaus Schmider, Der jugoslawische Kriegsschauplatz, S. $1009 \mathrm{f}$.

9 Korb, Im Schatten des Weltkriegs, S. 88.

10 OB Südost (Heeresgr. F) Generalfeldmarschall Maximilian von Weichs, H.Q., „Die große Absetzbewegung im Südosten“, Januar 1945, BArchF, RH 19-XI/86, S. 1.

11 Hermann K. Frank: Partisanenkampf in Albanien, in: Allgemeine Schweizerische Militärzeitschrift 120 (1954), S. 358. 
Der Sonderbeauftragte Südost, Hermann Neubacher, bestätigt diesen Eindruck:

Wenn je in einem Raume die Koordination der Außenpolitik notwendig war, dann war es der Balkanraum während des Krieges. Die neuen Grenzziehungen hatten die Verzahnungen der nationalen Siedlungsgebiete nicht verbessert. Die alten Gegnerschaften erhielten eine neue blutige Nahrung und zu den alten Verwicklungen trat die ganze Problematik der Besetzung durch auswärtige Mächte. ${ }^{12}$

Obwohl Verfolgungen, Plünderungen und Massaker vor neu gezogenen Grenzen nicht haltmachten, unterschieden sich Art und Ausmaß deutscher und italienischer Einflußnahme und Herrschaft in den drei Gebieten, dem NDH, Serbien (mit Nordkosovo) und „Großalbanien“ stark. Die jeweilige Art der Zusammenarbeit mit den drei Staaten beeinflusste auch die Art und Intensität der Gewaltanwendung und die Rekrutierungen für die Waffen-SS. Überall ähnlich gestalteten sich allerdings sowohl die Unüberschaubarkeit verschiedener Konflikte und Konfliktparteien in ethnisch stark gemischten Grenzregionen - als auch Kompetenzstreitigkeiten zwischen den beiden Besatzungsmächten selbst.

Nach der siegreichen Intervention auf dem Balkan mussten Hitler und Mussolini ihre politischen, militärischen und vor allem wirtschaftlichen Interessen neu diskutieren und die Aufteilung der Bodenschätze in den neu eroberten Gebieten regeln. Differenzen waren vorprogrammiert. ${ }^{13}$ Obwohl der Grenzverlauf teilweise noch bis weit ins Jahr 1942 Anlass zu Diskussionen gab, ${ }^{14}$ hatten die Deutschen die für sie am wichtigsten erscheinenden wirtschaftlichen Objekte und Zonen für sich reklamieren können: ${ }^{15}$ Serbien mit Nordkosovo kam unter deutsche Militärherrschaft. Denn die Zink- und Bleiminen in Trepça im Norden Kosovos, ein schon damals großes Fördergebiet, ehemals unter britischer Leitung, waren für Deutschland von größter Bedeutung. Die Deutschen verlangten auch die Förderwerke am Ljuboten. ${ }^{16}$ Die Kupfermine bei Bor sollte ebenfalls von den Deutschen ausgebeutet werden und bei der Chromerzförderung bei Jeserina wurde, obwohl das Gebiet zu Bulgarien kam, vertraglich festgelegt, dass

12 Neubacher, Sonderauftrag Südost, S. $127 \mathrm{f}$.

13 Vgl. Schmider, Partisanenkrieg, S. 33.

14 Vgl. bspw. deutsche Gesandtschaft Tirana, Schliep an das Albanische Ministerium für Auswärtige Angelegenheiten, 21.8.1944, A.Q.SH., V. 1944, D. 46, fl. 77. Siehe ausführlich zur deutschen und italienischen Besatzungspolitik im NDH: Sanela Schmid, Deutsche und italienische Besatzung im Unabhängigen Staat Kroatien 1941-45, Dissertation Universität Bern, unveröffentlicht.

15 Vgl. Schmider, Partisanenkrieg, S. 33.

16 Vgl. Zaugg, Albanische Muslime, S. 80. 
die Deutschen die Föderrechte behielten. Auch die Bahnlinie Zagreb (Agram) Belgrad - Niš war unter deutscher Kontrolle. ${ }^{17}$

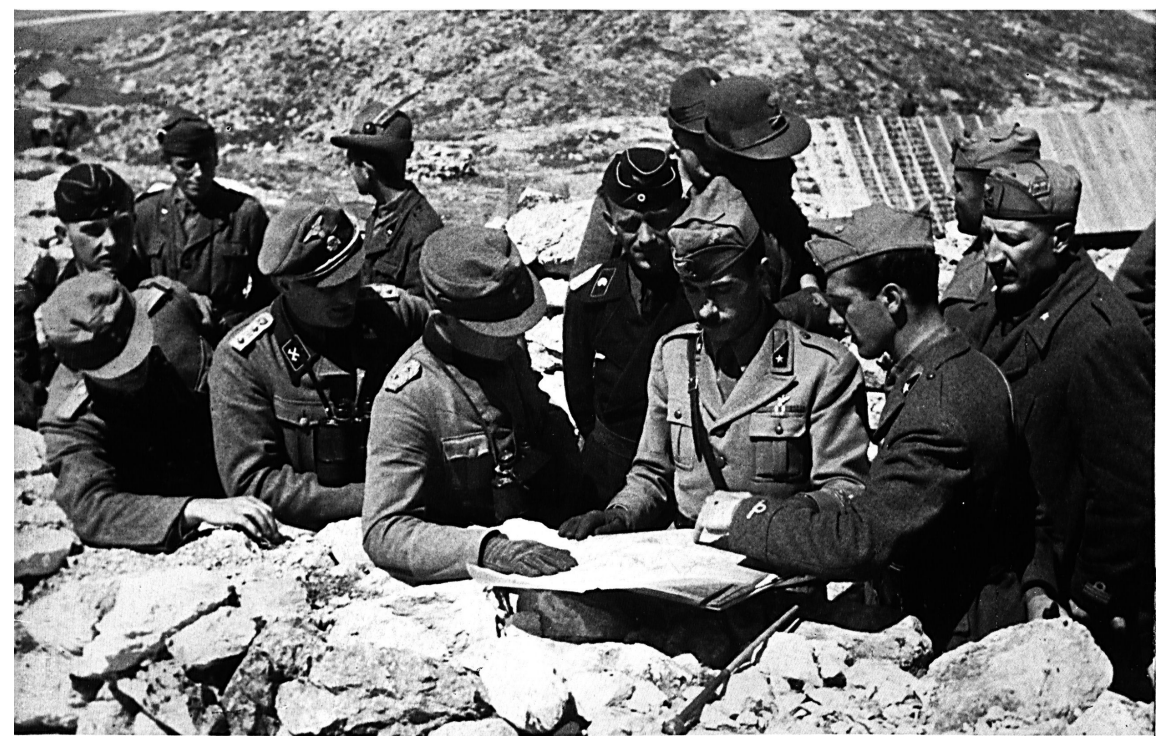

Abb. 1: „Lagebesprechung mit den italienischen Verbündeten und den Panzermännern des Heeres.“18

Mussolini, der sich mit dem weniger interessanten Teil Kosovos und den Gebieten bis nach Debar und Struga im heutigen Mazedonien sowie den kroatischen Küstengebieten zufrieden geben musste, forderte für Italien den Abbau der Bauxitvorkommen bei Mostar. ${ }^{19}$ Er war aber letztlich vor allem froh, seine wirtschaftlichen Interessen in Südosteuropa überhaupt weiterverfolgen zu können, wie Außenminister Galeazzo Cianos Tagebucheintrag vom 28. Juni 1940, zehn Monate vor dem Balkanfeldzug, zu entnehmen ist: „Es muss um jeden Preis ein Konflikt auf dem Balkan vermieden werden, der uns der wirtschaftlichen

17 Vgl. Schmider, Partisanenkrieg, S. 33. Die Ausbeutung dieser neuen Gebiete war jedoch von einem hohen Grad an Improvisation geprägt und auch Fehllieferungen im großen Stil kamen vor wie etwa folgende Begebenheit zeigt: Am 4.2.1942 meldete Berger an Himmler, dass vorerst zwar aus Kroatien kein Kupfer, dafür aber 20.000 kg Bronze geliefert würden. Die Bezahlung sollte in „Material“ erfolgen. Solche Händel sollten aber vor der Öffentlichkeit geheim gehalten werden. Berger an Himmler, betr. Bronze aus Kroatien, 4.2.1942, BArchB NS19/319, Bl. 14.

18 Nachlass Vopersal, BArchF, N 756/149a.

19 Vgl. Schmider, Partisanenkrieg, S. 33. Vgl. Zaugg, Albanische Muslime, S. 80. 
Ressourcen berauben würde.“20 Im Gegensatz zu Mussolini war Hitler an einer Zerschlagung Jugoslawiens nie interessiert gewesen. ${ }^{21}$ Denn bis März 1941 war Jugoslawien aus deutscher Sicht „ein leistungsfähiger, für die deutsche Kriegswirtschaft höchst wertvoller Handelspartner [gewesen]: Weizen, Mais, Fleisch, Fett, Ölsaaten, Hanf, Holz, Bauxit, Kupfer, Blei, Antimon, Molybdän usw. waren die kriegswirtschaftlich wertvollen Güter, die Jugoslavien [sic] im Warenaustausche anzubieten hatte.“22

Zuerst schien es, als ob die Lage nach einem kurzen Krieg von nur elf Tagen rasch unter Kontrolle zu bringen sei: Nur vier deutsche Reservedivisionen, die 704., 714., 717. und 718. Infanterie-Division, welche sich alle noch in Aufstellung befanden, wurden zur Sicherung im NDH und Serbien zurückgelassen. ${ }^{23}$ Die 704. Infanterie-Division war zuerst im Gebiet Priboj, anschließend im Raum Valjevo und danach in der Gegend von Belgrad stationiert. Die 714. InfanterieDivision wurde zuerst bei Bor und Palanka/Plankenburg sowie südlich der Velika Plana eingesetzt. Bereits im Herbst 1941 fanden in Südserbien bei Kragujevac, Užice und Čačak heftige Kämpfe vor allem gegen Četnik-Partisanen statt. Die 717. Infanterie-Division agierte im Raum Kraljevo, Kragujevac und Mitrovica und verübte während dieser Zeit die zwei größten Massaker in Südosteuropa während Zweiten Weltkriegs: In Kraljevo und Kragujevac töteten Soldaten des Infanterieregiments 749 der 717. Infanterie-Division Mitte Oktober 1941 4.000 Zivilisten als Vergeltungsmaßnahme. ${ }^{24}$ Im Unterschied zu „Großalbanien“ und dem NDH bildete aber Serbien in großen Teilen ein homogenes Siedlungsgebiet. Lediglich in oben genannten Gebieten in Südserbien, zu welchen auch die nördlichen Teile Kosovos und Teile des Sandžaks zählten, sowie in der Vojvodina (mit der Bačka und dem Banat) lebten größere, nicht-serbische Bevölkerungsgruppen. ${ }^{25}$ Genau in diesen drei Teilen wurde seit spätestens 1942 intensiv für die Waffen-SS geworben.

20 Ciano, Tagebuch, 28.6.1940, S. 253. Zum gebotenen kritischen Umgang mit den Tagebüchern Cianos, die in dieser Arbeit als Abgleich zu anderen Quellen dienen werden, vgl. Tobias Hof, Die Tagebücher von Galeazzo Ciano, in: Vierteljahreshefte für Zeitgeschichte 4(2012), S. 507-527.

21 Vgl. Neubacher, Sonderauftrag Südost, S. 129.

22 Ebd. S. 129.

23 Vgl. Klaus Schmider, Der jugoslawische Kriegsschauplatz, S. 1012f.

24 Vgl. Klaus Schmider, Auf Umwegen zum Vernichtungskrieg? Der Partisanenkrieg in Jugoslawien, 1941-1944, in: Rolf-Dieter Müller, Hans Eriche Volkmann (Hgg. im Auftrag des Militärgeschichtlichen Forschungsamts), Die Wehrmacht. Mythos und Realität, S. 901 . Seit 1942 war die 717. Infanterie-Division in größeren Unternehmen gemeinsam mit der 7. SS-Freiwilligen-Division „Prinz Eugen“ eingesetzt. Vgl bspw. Rolf Michaelis, Die Gebirgs-Divisionen der Waffen-SS, Erlangen: Miachelis-Verlag 1994, S. 69.

25 Vgl. Schmider, Partisanenkrieg, S. 32. 
Rasch zunehmende Überfälle der sich bildenden Partisaneneinheiten, sowohl auf kommunistischer als auch monarchistischer Seite, stellten die Wehrmacht wie auch die deutsche Verwaltung vor große Herausforderungen. Die zahlreichen, bereits in den ersten Tagen des neugegründeten NDH einsetzenden Massaker von Ustaša-Angehörigen gegen Serben, Roma und Juden provozierten spontane Aufstände von Seiten der bedrohten Bevölkerungsteile, insbesondere von serbischer Seite. Gleichzeitig rief auch die Kommunistische Partei Jugoslawiens dazu auf, das neue Ustaša-Regime zu bekämpfen. Bereits drei Wochen nach dem Balkanfeldzug wurde die Osvobodilna Fronta (Befreiungsfront) in Ljubljana gegründet, eine antifaschistische Dachorganisation, die 15 politische Parteien und Zivilorganisationen vereinte. ${ }^{26}$ Bereits im Mai 1941 wurde unter dem Kommando der jugoslawischen Exilregierung in London die erste Guerilla-Einheit in Serbien ins Leben gerufen. Dies war der Beginn der so genannten monarchistisch ausgerichteten Jugoslovenska Vojska u Otadžbini (Jugoslawischen Armee im Vaterland), besser bekannt als Četnik-Bewegung, unter dem Oberkommando des ehemaligen serbischen Offiziers Dragoljub Draža Mihailović. ${ }^{27}$ Nur wenige Wochen später, am 22. Juni 1941, bildeten auch die Kommunisten ihre erste Partisaneneinheit. Am 4. Juli rief die Kommunistische Partei Jugoslawiens zum bewaffneten Widerstand gegen die italienischen Streitkräfte in allen ehemaligen jugoslawischen Gebieten auf. Dieser dehnte sich später auch auf die von Deutschland besetzten Zonen aus. ${ }^{28}$ In Serbien galten die höchsten deutschen Sühnekontingente mit $50 \mathrm{zu}$ erschiessenden Geiseln für einen verletzten und 100 für einen getöteten deutschen Soldaten. ${ }^{29}$

Unter zunehmendem Druck rasch anwachsender kommunistischer Partisanenverbände näherten sich die Četnik-Verbände den Deutschen an, so beispielsweise in Leskovac und Niš. ${ }^{30}$ Da die Deutschen den serbischen Monarchisten Waffen und Munition lieferten, ${ }^{31}$ ließen die Briten im Juni 1944 über die Nachrich-

26 Obwohl die Osvobodilna Fronta zuerst aus Vertretern verschiedener politischer Parteien bestand, war der Einfluss der kommunistischen Partei letztlich entscheidend.

27 Später kollaborierte jedoch ein Großteil der Četnik-Bewegung sporadisch oder auch ständig mit den deutschen Besatzern.

28 Branko Petranović, Istorija Jugoslavije, II, Beograd: Nolit 1988, S. 52-153.

29 Vgl. Schmider, Partisanenkrieg, S. 64 und Calic, Geschichte Jugoslawiens, S. 142f. Im Gebiet des NDH waren die Deutschen bald gezwungen, die Sühnekontingente anzupassen auf 1:10 und Erschießungen von Geiseln nur in Absprache mit den kroatischen Behörden vorzunehmen, Schmider, Auf Umwegen zum Vernichtungskrieg?, S. 905.

30 Vgl. Chef des Generalstabs Militärbefehlshaber Südost, von Geitner an Deutschen Bevollmächtigen General in Albanien, 20.2.1944, BArchF, RW40/85, Bl. 96.

31 Vgl. Meldekopf Rozhaj an Ltn. Heckner, Scheiben betr. „Waffen und Munition für Četniks“, 8.9.1944, BArchF, 3-21/2, Bl. 131. 
tenagentur Reuters verlauten, dass sie ihre Unterstützung der Četnik-Verbände eingestellt hätten und nur noch die Kommunsiten unterstützen würden. ${ }^{32}$ Die Četniks blieben aber auch für die Deutschen unsichere Verbündete. Ein Befehl der Abteilung Ia des Militärbefehlshabers Südost an die Höheren SS- und Polizeiführer Südosteuropas warnte davor, dass diese bei der ersten sich bietenden Gelegenheit wieder gegen die deutschen Truppen kämpfen würden. ${ }^{33}$ Denn obwohl einige Četnik-Gruppen mit den Deutschen zusammenarbeiteten, ermordeten andere Četniks, die weiterhin die Zusammenarbeit mit den Deutschen verweigerten, Kollaborateure - so beispielsweise den Bürgermeister von Kragujevac. ${ }^{34}$ Andere wiederum, wie die Anhänger Radoslav Rade Radićs propagierten eine Zusammenarbeit mit den kommunistischen Partisanen, da sie ohne die Unterstützung der Briten zu schwach für den Kampf waren. Auf deutscher Seite war man sich sicher, dass zwischen Tito und dem Ministerpräsidenten der Exilregierung, Ivan Šubašić, eine Besprechung stattgefunden hatte; auch zwischen Mihailović und Šubašić vermutete man eine ebensolche. ${ }^{35}$

Aufgrund ausbleibender Erfolge und dem Anwachsen gegnerischer Formationen lockerte der Oberbefehlshaber Südost, Generaloberst Alexander Löhr, auf Befehl Hitlers am 22. Dezember 1943 die bisher geltenden „Sühne-, Straf-, und Vergeltungsmaßnahmen“. Die Begründung lautete:

Das Verfahren, nach dem Überfall oder Sabotageakt aus der näheren Umgebung des Tatortes wahllos an Personen und Wohnstätten Sühnemaßnahmen zu vollziehen, erschüttert das Vertrauen in die Gerechtigkeit der Besatzungsmacht und treibt auch den loyalen Teil der Bevölkerung in die Wälder. ${ }^{36}$

Doch für den serbischen Raum sind auch nach Lockerung der Sühnekontingente zahlreiche Festnahmen und Erschießungen von ganz unterschiedlichen Gegnern belegt. So wurden beispielsweise kurz darauf, Anfang Februar 1944, bei dem „Säuberungsunternehmen gegen [ein] Kommunistendorf“ südlich von Niš 16 Kommunisten erschossen und 30 Verdächtige festgenommen, am selben Tag wurden weiter nördlich im Banat als Vergeltung eines Anschlags auf einen

32 Vgl. Junker an Neubacher und Generalkonsulat Tirana, 21.6.1944, PAAA R27305.

33 Vgl. Militärbefehlshaber Südost, Abt. 1a, i.V. General der Artillerie an Höhere SS- und Polizeiführer, 11.2.1944, BArchF, RW40/85, Bl. 59.

34 Vgl. Chef des Generalstabs Militärbefehlshaber Südost, von Geitner an Heeresgruppe F, 12.2.1944, BArchF, RW40/85, Bl. 65.

35 Vgl. Junker an Tirana, 20.6.1944, PAAA R27305. Das Treffen zwischen Tito und Šubašić fand am 16. Juni 1944 auf der Insel Vis statt.

36 OB Südost, Generaloberst Alexander Löhr, Abschrift „Sühnemaßnahmen“, 22.12.1943, BArchF, RW 40/89, S. 3. 
deutschen Zollgrenzschutz 15 kommunistische „Sühnegefangene“ erschossen. ${ }^{37}$ Beim Unternehmen „Treibjagd“, welches ebenfalls im Februar 1944 durchgeführt wurde, wurden gleich zu Beginn 70 Četniks und ihr Anführer festgenommen. ${ }^{38}$

Die deutsche Herrschaft in Albanien unterschied sich deutlich von derjenigen in Serbien und Kroatien und lässt sich in zwei Stufen gliedern. Bis 1943 war der Einfluß deutscher Dienststellen beschränkt auf die an "Großalbanien” angrenzenden Gebiete, Norkosovo und Sandžak. Die übrigen, vor allem von Albanern bewohnten Gebiete Mittel- und Südkosovos, kamen nach dem Balkanfeldzug 1941 als „neualbanische“ Gebiete zu „Großalbanien“ unter italienischer Herrschaft. Auch hier ist der Einfluss der Achsenmächte auf die Verschärfung interethnischer Konflikte unverkennbar. Das „Laisser faire“ der beiden faschistischen Staaten führte dazu, dass in den Grenzgebieten in und um Kosovo Tausende Menschen auf der Flucht waren und Übergriffe zunahmen. ${ }^{39}$ Doch auch in „Altalbanien“ war die Situation instabil bis prekär: Hungersnöte mussten etwa durch Ernten aus dem Kosovo entschärft werden. ${ }^{40}$

Flüchtlingsströme aus kroatischen, bulgarischen und ungarischen Gebieten verschärften die bereits angespannte Situation gerade in Serbien, aber auch in Bosnien zusätzlich. ${ }^{41}$ Aus dem nun zu „Großalbanien“ gehörenden Teil Kosovos strömten verfolgte serbische Familien. ${ }^{42}$ Diese waren in der Zwischenkriegszeit oft unter Zwang in der Region angesiedelt worden. Nun verließen sie ihre Häuser, ihren Besitz und ihre Grundstücke, um nicht ermordet zu werden. Bereits nach seiner Reise durch albanisch bewohnte Gebiete beidseits der Demarkationslinie stellte Volkstumsreferent Otto Feninger im Herbst 1941 fest,

dass die Arnauten serbische Ansiedler aus dem Arnautengebiet durch Verbrennung der Häuser, in manchen Fällen ganzer Dörfer vertrieben haben. Diese Maßnahmen haben jedoch diesseits der deutsch-italienischen Demarkationslinie beiweitem [sic] nicht derartige

37 Chef des Generalstabs Militärbefehlshaber Südost, von Geitner an Heeresgruppe F, 12.2.1944, BArchF, RW40/85, Bl. 65.

38 Chef des Generalstabs Militärbefehlshaber Südost, von Geitner an Heeresgruppe F, 18.2.1944, BArchF, RW40/85, Bl. 88.

39 Vgl. Rodogno, Nuovo ordine, S. 353. Vgl. Zaugg, Albanische Muslime, S. 152-154.

40 Vgl. Zaugg, Albanische Muslime, S. 114.

41 Vgl. Schmider, Partisanenkrieg, S. 33.

42 Vgl. Volkstumsreferent Dr. Feninger beim Bevollmächtigten des AA in Belgrad, Bericht „über die reise in das Arnautengebiet von Kosovska Mitrovica und Novi Pazar“, 15.-26.10.1941, PAAA, R261153, Bl. 36. Aufgrund der chaotischen Situation in diesem Gebiet waren keine genauen Angaben über die Anzahl dieser serbischen Flüchtlinge möglich. Vgl. ebd. 
Ausmaße erreicht, wie in den von Italien besetzten Gebieten, wo während der Fahrt von Mitrovica nach Pec [sic] eine große Zahl brennender serbischer Dörfer und serbische und montenegrinische Flüchtlinge in großer Zahl auf den Straßen beobachtet wurden. ${ }^{43}$

Mehr als zwei Jahre später, im Februar 1944, war die Situation unverändert, wie Geitners Meldung an die Heeresgruppe F zu entnehmen ist: „Seit Januar im Gang befindliche Flüchtlingbewegung serb[ischer] Kolonisten aus dem alb[anischen] Kosovo dauert an. Bisher haben ca. 1.800 Personen die Grenze überschritten, 600 weitere sind für die nächsten Tage angekündigt. " ${ }^{44}$ In den dreieinhalb Jahren (April 1941 bis Oktober/November 1944) deutscher und italienischer Besatzung der Kosovoregion und angrenzender Gebiete sollen bis zu 40.000 Serben aus dem Gebiet geflohen sein. ${ }^{45}$

Ähnlich große Menschenmassen waren im NDH in Bewegung, wo in den größeren Städten Bosniens zehntausende muslimischer Flüchtlinge auf engstem Raum lebten und viele von ihnen an Hunger und Krankheiten starben. ${ }^{46}$

\subsection{Instrumentalisierung interethnischer Gewalt als politisches Prinzip der Besatzungsmächte}

So unterschiedlich sie in einigen Aspekten auch sein mögen, allen vier hier vorgestellten Divisionen liegt ein wesentliches Element zugrunde: Sie wurden aufgrund ethnischer bzw. ethnisch-religiöser Prinzipien aufgebaut. Ethnische Zuordnung und Zuschreibungen spielten für die Rekrutierung in die Waffen-SS im Speziellen und die Politik der Achsenmächte in Südosteuropa im Allgemeinen eine zentrale Rolle. ${ }^{47}$ Ethnizität ist das verbindende und paradoxerweise gleichzeitig trennende Element der südosteuropäischen Waffen-SS-Divisionen. Die Instrumentalisierung des Ethnischen darf aber nicht darüber hinwegtäuschen, dass in allen vier Divisionen auch Angehörige anderer Ethnien bzw. Religionen kämpften. So sind sowohl

43 Volkstumsreferent Dr. Feninger beim Bevollmächtigten des AA in Belgrad, Bericht „über die Reise in das Arnautengebiet von Kosovska Mitrovica und Novi Pazar“, 15.-26.10.1941, PAAA, R261153, Bl. 53.

44 Chef des Generalstabs Militärbefehlshaber Südost, von Geitner an Heeresgruppe F, 14.2.1944, BArchF, RW40/85, Bl. 70.

45 Vgl. Elsie, Historical Dictionary of Kosovo, S. 252.

46 Vgl. Brief Nr. 17 an Ernst von Weizsäcker, 9.6.1942, in: Gerhard Höpp (Hg.), Die Mufti-Papiere. Briefe, Memoranden, Reden und Aufrufe Amin al-Husainis aus dem Exil, 1940-1945, Berlin: Klaus Schwarz 2001, S. 43. Vgl. auch Betr. „Von Reichsführer-SS befohlene Sammlung zur Behebung der Not der muselmanischen Bevölkerung im bosnischen Raum“, Berger an Himmler, 12.1.1944, BArchB NS19/2601, Bl. 165.

47 Vgl. Casagrande, Volksdeutsche SS-Division, S. 325. 
für die „Handschar“ als auch für die „Skanderbeg“ Katholiken nachgewiesen; zu den Hilfstruppen gehörten auch orthodoxe Christen. ${ }^{48}$

Durch die bewusste Betonung ethnischer Merkmale schürten die Vertreter der Waffen-SS und der deutschen Verwaltung vor Ort gezielt bereits schwelende Konflikte zwischen verschiedenen Volksgruppen. Zur Definition von solchen Konflikten, welche entlang ethnischer Grenzen verlaufen, sind in den letzten Jahren verschiedene Begriffe entwickelt worden. So beispielsweise der Begriff des „ethnischen“, „ethnisierten“ oder „ethnonationalen“ Konflikts. ${ }^{49}$ Der hier verwendete Terminus des „interethnischen“ Konflikts legt den Fokus auf zwischen zwei oder mehreren Ethnien ausgetragene Auseinandersetzungen, hier vor allem zwischen orthodoxen Serben bzw. Montenegrinern (und in Bosnien lebenden orthodoxen Serben), katholischen Kroaten (und in Bosnien lebenden Katholiken) sowie der in Bosnien, Kosovo und Grenzgebieten wie Südserbien oder Nordalbanien lebenden Muslimen.

Allerdings soll bereits hier darauf hingewiesen werden, dass längst nicht jede Gewaltanwendung einen ethnischen Hintergrund hatte. Wie die involvierten Parteien, ihre komplexen Kollaborationen und Brüche darin zeigen, weist Gewalt in solchen Zusammenhängen stets einen multikausalen Charakter auf. ${ }^{50}$ Diese Multikausalität von Gewaltvorkommen und -anwendungen gleicht einem dicht gewobenen Netz, dessen Stränge hinreichend entflochten und beleuchtet werden müssen, um die Rekrutierungen für die Waffen-SS in diesen Gebieten erklären und verstehen $\mathrm{zu}$ können. ${ }^{51}$

Zahlreiche Studien zu Südosteuropa greifen das Spannungsfeld des Zusammenlebens verschiedener Ethnien, der Abgrenzung voneinander, der Instrumentalisierung ethnischer Merkmale und daraus resultierender Konflikte auf. ${ }^{52}$ Eva

48 Vgl. Zaugg Interview mit Tush Mark Ndou 7.6.2017. Vgl. auch Zaugg, Albanische Muslime, S. 233-234. Siehe zur bis heute anhaltenden Diskussion um ethnische Aspekte in den Konflikten Südosteuropas bspw. Hannes Grandits: Wie die Gewalt der Jugoslawienkriege konzeptionalisieren? Wissenschaftliche Ansätze seit den 1990er Jahren, Südosteuropa. Zeitschrift für Politik und Gesellschaft 04/2013, S. 578-594.

49 Vgl. beispielsweise Korb, Im Schatten des Weltkriegs, S. 43. Vgl. Sundhaussen, Ethnonationale Gewalt, S. 37, 39f. Vgl. Wolfgang Höpken, Michael Riekenberg (Hgg.), Politische und ethnische Gewalt in Südosteuropa und Lateinamerika, Wien etc.: Böhlau 2001.

50 Hier sei auf Christian Gerlachs Studie, Extremly Violent Societies. Mass Violence in the Twentieth-Century World, Cambridge/New York 2010, S. 7-10, 342 verwiesen.

51 Vgl. Zaugg, Albanische Muslime, S. 180.

52 Für verschiedene Zeiträume und Regionen beispielsweise: Calic, Marie-Janine, Der Jugoslawienkrieg der 1990er Jahre: Theorie des ethno-nationalistischen Krieges, in: Thomas Jäger; Rasmus Beckmann (Hg.), Handbuch der Kriegstheorien, Wiesbaden 2011, S. 448-456. Höpken, Riekenberg (Hgg.), Politische und ethnische Gewalt. Sundhaussen, Dorf, Religion und Nation, Über den Wandel 
Anna Frantz fächert diese Wahrnehmungsmuster von „Eigenem“ und „Fremden“ für die Region Kosovo in spätosmanischer Zeit auf. Sie betont, dass zwar Zugehörigkeiten zu Verwandtschafts-, Stammes- oder Berufsgruppen eine wichtige Rolle spielten, dass aber innerhalb der soziokulturellen Verortung neben lokalen und regionalen Aspekten vor allem religiöse und ethnische Abgrenzungen prägend waren. ${ }^{53}$ Sundhaussen hingegen schreibt, dass die Größen "Dorf” bzw. "Stamm” für die lokale Bevölkerung zentrale Bezugspunkte bildeten und das Zusammenleben regelten und somit auch als System von In-und Exklusion dienten. Ein- und Ausschluss aufgrund der Religionszugehörigkeit versteht er eher als Folgen des Nationsbildungsprozesses und der damit einhergehenden Umwandlung von Territorial- und Nationalkirchen. ${ }^{54}$ Vesna Pešić beispielsweis untersuchte die Instrumenstalisierung des Ethnischen, welche sie als eine "Mobilisierung des Ethnischen” bezeichnet, für die Zeit vor den Jugoslawienkriegen der 1990er Jahre. Sie schlägt für dieses Vorgehen folgende Definition vor, die auch auf die Zustände in Bosnien, Kroatien und Albanien und innerhalb "volksdeutscher" Siedlungsgebiete zutreffen: "Ethnic mobilization is a state-building movement of a people led by an authoritarian leader who had succeeded in assuming control over the state apparatus, the media, the Church and the cultural elite, the involvement of which is prerequisite for achieving the emotional, cultural and political homogenization of the nation's awareness of the common enemy war should be waged against.“55

Vor allem in ethnisch stark durchmischten Gebieten Südosteuropas, namentlich den beiden Hauptregionen für die Rekrutierungen der „Handschar“ und der „Skanderbeg“, kam es nicht nur während des Zweiten Weltkriegs, sondern während des gesamten 20. Jahrhunderts immer wieder zu Übergriffen und gewalttätigen Aussschreitungen. Auch in Friedenszeiten waren die jeweiligen Minderheiten dieser Gebiete sowohl sozialer Benachteiligung ${ }^{56}$ als auch physischer und psychischer

vorgestellter Gemeinschaften im Balkanraum, Journal of Modern European History 1(2011), S. 87116. Imanuel Geiss, Der Balkan als historische Konfliktregion, in: Elvert, Jürgen (Hg.), Der Balkan: Eine europäische Krisenregion in Geschichte und Gegenwart, Stuttgart: Franz Steiner 1997, S. 21-36. Weitere Publikationen zu diesen Themenfeldern finden sich in der Bibliographie.

53 Vgl. Eva Anne Frantz, Gewalt und Koexistenz. Muslime und Christen im spätosmanischen Kosovo (1870-1913), München: De Gruyter/Oldenbourg 2016, S. 193. Vgl zur Definition von „Eigenem“ und „Fremdem“ detailliert Casagrande, Volksdeutsche SS-Division, S. 39, $48 \mathrm{f}$.

54 Vgl. Holm Sundhaussen, Dorf, Religion und Nation., S. 88, 90, $96 \mathrm{f} ., 102 \mathrm{f}$.

55 Vesna Pešić, Ethnic Mobilization in Serbia, Bozen: Europäische Akademie 2007, S. 3.

56 Zur Definition struktureller Gewalt vgl. Johan Galtung, Gewalt, Frieden und Friedensforschung, in: Dieter Senghaas (Hg.), Kritische Friedensforschung, Frankfurt M. 1971, S. 55-104. Bis heute wird dieser Begriff insbesondere aber seine Trennschärfe und daraus resultierende Missverständnisse in der Forschung diskutiert. Er soll hier nicht wie etwa von Michael Riekenberg 
Gewalt ausgesetzt: Dies reichte vom Schulausschluss der Kinder, ${ }^{57}$ der Benachteiligung bei der Arbeitsuche, dem Verbot, die eigene Sprache zu sprechen bzw. zu unterrichten, über überhöhte Steuerabgaben, Drohungen, Erpressungen, Viehdiebstahl, Druck oder Zwang, einen bestimmten Glauben anzunehmen, Umsiedlungen, bis hin zur physischen Vernichtung ganzer Dörfer und ihrer Bewohner. ${ }^{58}$

In ihrer Untersuchung unterscheidet Frantz zur klareren Abgrenzung zwischen traditionellen Formen von Alltagsgewalt und religiös bzw. ethnisch konnotierten Spannungen und Konflikten. ${ }^{59}$ Doch letztlich lässt sich auch mancher Akt von Alltagsgewalt vor einem ethnischen Hintergrund lesen. Nach Sundhaussen wurden die jeweiligen Minderheiten aufgrund ihrer Volkszugehörigkeit als „minderwertig“, „bedrohlich“ und „fremd“ inszeniert und schließlich auch so wahrgenommen - mit dem Ziel, ein bestimmtes Gebiet, eine Region „ethnisch zu säubern“. Durch erzwungene Anpassung, beispielsweise der Unterdrückung der eigenen Sprache und durch Animation zur Flucht anhand von Gräuelpropaganda, Diskriminierung, Demütigung, sexueller Gewalt oder der Zerstörung wirtschaftlicher Grundlagen sollten diese Menschen assimiliert bzw. vedrängt werden. Wer dann immer noch zögerte, dem drohte eine gewaltsame Vertreibung oder Ermordung. ${ }^{60}$ Gerade im südosteuropäischen Raum hatten Versuche „ethnischer Entflechtung“ eine lange Tradition: „Ebenso wichtig wurde, dass im Labor Balkan seit längerem die Politik-Alternative der ,Säuberung‘ von einer bloßen Begleiterscheinung von Kriegen zur großräumig angelegten Friedensregelung ausdifferenziert worden war. Bereits Lausanne [Vertrag von Lausanne 1923F.Z.] war die Summe eines Jahrhunderts organisierter Gewaltpolitik.“61 Die Politik „ethnischer

verwendet werden. Dieser interpretierte Galtungs Definition dahingehend, „dass es eine Gewalt gebe, die allein als Struktur bestehe und frei sei vom Handeln sozialer Akteure“ https://zeithisto rische-forschungen.de/1-2008/id=4655 (Stand: 20.1.2019) Vielmehr soll auf jene Struktur verwiesen werden, die von Akteuren geschaffen, zur Vernachlässigung und Behinderung der Entwicklung anderer führt.

57 Eine Methode, die nach dem Ersten Weltkrieg, im Zweiten Weltkrieg, später auch während der Konflikte der 1990er Jahre angewendet wurde.

$58 \mathrm{Vgl}$. Zaugg, Albanische Muslime, S. 148f. Die Verfasserin untersucht zurzeit in ihrem Habilitationsprojekt die Frage nach einer ,longue durée‘ von Gewalt und kriegsversehrten Gesellschaften in Südosteuropa. Affiliiert an der Universität Bern/finanziert durch den Schweizerischen Nationalfonds.

59 Frantz, Gewalt und Koexistenz, S. 276-304.

60 Vgl. Holm Sundhaussen, Ethnische Säuberung, in: Sundhaussen, Holm/Clewing, Konrad (Hgg.), Lexikon zur Geschichte Südosteuropas, Wien 2016, S. 310.

61 Vgl. Schwartz, Michael, Ethnische „Säuberungen“ in der Moderne: globale Wechselwirkungen nationalistischer Gewaltpolitik im 19. und 20. Jahrhundert, München: Oldenbourg 2013, S. 236. 
Säuberung“ von bestimmten Gebieten wurde somit auch Teil internationaler Friedensverträge. ${ }^{62}$

$\mathrm{Zu}$ den Hauptopfern der Gewalt im Zweiten Weltkrieg - aufgerieben und zermalmt zwischen verschiedensten Kräften und Gruppierungen - zählte die unbewaffnete Bevölkerung aller Ethnien, nämlich Frauen, Kinder und alte Menschen. Sie waren es, die die kleinste Mobilität aufwiesen und sich aufgrund physischer Bedingungen und mangelnder Ausrüstung und Ausbildung am schlechtesten verteidigen konnten.

Gewalt an ethnischen Minderheiten wurde einerseits von Teilen der machthabenden Elite aktiv unterstützt und von Teilen der lokalen Bevölkerung mitgetragen. Andererseits hegten auch die Deutschen konkrete Pläne, wer wohin zu deportieren sei. Bereits am 4. Juni 1941 versammelte der deutsche Gesandte in Agram Siegfried Kasche 18 Vertreter der kroatischen Regierung, um mit ihnen großangelegte Umsiedlungspläne zu besprechen. So sollten 180.000 Slowenen nach Kroatien und rund 200.000 von Kroatien nach Serbien umgesiedelt werden. ${ }^{63}$ Korb geht davon aus, dass solchen Vertreibungen, die das erste Kapitel der von der Ustaša verübten Massengewalt bildeten, rund 300.000 Menschen zum Opfer fielen. ${ }^{64}$ Doch nicht nur die Ustaša-Elite, auch der bekannteste ČetnikFührer, Draža Mihailović, war als Monarchist Anhänger eines ethnisch reinen „Großserbiens“ und somit Befürworter von Umsiedlungen bzw. Vertreibungen von Minderheiten und sogenannten „anationalen Elementen“. Rund 2,7 Millionen Menschen sollten seiner Meinung nach umgesiedelt werden: Albaner, Kroaten, bosnische Muslime und „Volksdeutsche“. Diese Absichten zogen sich, trotz Kaschierung in der Öffentlichkeit, wie ein roter Faden durch die internen Dokumente der Četnik-Bewegung. ${ }^{65}$ Und schließlich waren auch die „neualbanischen“/kosovarischen Eliten mit dem Versprechen eines „ethnisch reinen“ „Großalbaniens“ für die Zusammenarbeit mit den Deutschen und den Italienern gewonnen worden. ${ }^{66}$

War es nach dem Ersten Weltkrieg im Ersten Jugoslawien vor allem die serbische Seite gewesen, die durch die Ansiedlung serbischer und montenegrinischer Bauern in albanisch bewohnten Gebieten wie dem Sandžak und Kosovo versucht hatte, den Anteil orthodoxer Bewohner in dieser Region zu erhöhen,

62 So ist auch der Vertrag von Dayton aufgrund der Legalisierung so genannter „gesäuberter“ Gebiete bis heute umstritten.

$63 \mathrm{Vgl}$. Korb, Im Schatten des Weltkriegs, S. 169.

64 Vgl. ebd.

65 Vgl. Sundhaussen, Geschichte Serbiens, S. 320-322.

66 Vgl. Brief von Pistor an Vopersal, undatiert, BArchF, N 756/182b. 
so verfolgten seit der Schaffung „Großalbaniens“ 1941 vor allem „neualbanische“ Machthaber die Politik „ethnischer Flurbereinigung“. ${ }^{67}$

Doch auch italienische und deutsche Dienststellen unternahmen Anstrengungen, durch Umsiedlungen und Deportationen bestimmte Bevölkerungsgruppen zufriedenzustellen und, wie sie betonten, Konflikte zu entschärfen. Im Gebiet des ehemaligen Sandžak von Novi Pazar wurde bereits im Herbst 1941 von deutschen Stellen die Aussiedlung albanischer Bevölkerungsteile diskutiert, da es seit April immer wieder zu gewaltsamen Ausschreitungen zwischen Serben und Albanern gekommen war. Die Besatzungmächte sahen in der geplanten Aussiedlung der Albaner in den italienisch besetzten Teil Kosovos eine Möglichkeit, die Gewalt einzudämmen. ${ }^{68}$ Allerdings warnte der deutsche Diplomat Peter Pfeiffer, ein Kenner der lokalen Verhältnisse, das Auswärtige Amt vor solchen Aussiedlungsaktionen, denn sie würden in den Augen der Albaner die „serbischen Kolonisierungsversuche der letzten Jahrzehnte“ zementieren und das Vertrauen in die Deutschen schädigen. ${ }^{69}$ Obwohl Pfeiffer selbst in diesen Umsiedlungsplänen auch Vorteile erkannte, riet er dringend davon ab, solche Absichten bei offiziellen Stellen zu erwähnen; denn sonst wäre es mit der „Ruhe in den Balkanländern“ endgültig vorbei. $^{70}$ Zum gleichen Zeitpunkt warnte auch Volkstumsreferent Otto Feninger nach einer „Reise in das Arnautengebiet von Kosovska Mitrovica und Novi Pazar“ vor Umsiedlungen und einer neuerlichen Veränderung der Demarkationslinie. Allerdings vertrat er die Sichtweise der Serben, denn „der völlige Verlust des Kosovo, das den Serben als alter Siedlungsboden und heilige Stätte schwerster opferreicher Schlachten gilt, wäre dem serbischen Volk gleichbedeutend mit der völligen Aufgabe seiner nationalen Existenz. ${ }^{\text {"71 }}$

67 Vgl. Schmitt, Kosovo, S. 207 f.

68 In einem Schreiben von Otto von Erdmannsdorf war von rund 100.000 Albanern die Rede, die von Südwestserbien nach „Großalbanien“ umgesiedelt werden sollten. Vgl. Auswärtiges Amt, Otto von Erdmannsdorff an Generalkonsulat Tirana, 15.11.1941, PAAA, Altes Amt, Tirana 4/3.

69 Generalkonsulat Tirana, Peter Pfeiffer an AA, 17.11.1941, PAAA, R261153, Bl. 81.

70 Ebd.

71 Volkstumsreferent Dr. Feninger beim Bevollmächtigten des AA in Belgrad, Bericht ,über die Reise in das Arnautengebiet von Kosovska Mitrovica und Novi Pazar“, 15.-26.10.1941, PAAA, R261153, Bl. 61. Nicht nur für den Norden, auch im Süden Albaniens war drei Jahre später ein ähnliches Umsiedlungsvorhaben vorgesehen. Rund 20.000 albanische Flüchtlinge aus der griechisch-albanischen Grenzregion Tsamouriá/Çamëria hatten sich in Filiates eingefunden. Diese sollten nun nach Südalbanien umgesiedelt werden. Auf der anderen Seite der Grenze sollte die griechisch-orthodoxe Bevölkerung gesammelt und nach Tsamouriá/Çamëria deportiert werden. Vgl. Junker an Schliep, 21.8.1944, PAAA, R27305. Es verwundert nicht, dass sich genau aus dieser Region zahlreiche Albaner für die Waffen-SS und lokale Polizeiverbände meldeten. Vgl. Arkivi i Kosoves, fondi: Dosjet e robërve të luftës në Gjermani, viti 1940-1945, kutia nr. 1. 
Hinter der Instrumentalisierung bereits bestehender interethnischer Konflikte stand aber auch die Absicht der Besatzer, die eigene Gewaltanwendung als Konfliktregulativ im Sinne einer westeuropäischen Ordnung zu präsentieren: „Die Taten der Ustascha [und anderer Gruppen] wurden als pittoresk-exotische Akte entfesselter, blutiger Gewalt gedeutet.“72 Diese Taten, die nach Ansicht der deutschen und italienischen Betrachter auf Emotionen und Affekten basierten, waren dementsprechend nicht Resultat rationaler Überlegungen. ${ }^{73}$ Aus dieser Perspektive betrachtet sollte die Gewalt der Besatzungsmächte die Konnotation einer überlegten, gut organisierten Gewalt erhalten - was mitnichten der Fall war. ${ }^{74}$ Doch nicht nur von außen, auch im Innern förderten die verschiedenen rivalisierenden Kräfte die Konstruktion eines kriegerischen Balkans. Denn jede Partei hegte das Interesse, die anderen Parteien in einem möglichst unzivilisierten Licht erscheinen zu lassen. ${ }^{75}$

Entsprechend versuchten lokale Eliten und deutsche sowie italienische Besatzer nicht, Unterschiede auszugleichen und Ähnlichkeiten zwischen den Volksgruppen $\mathrm{zu}$ betonen. Vielmehr wurden bereits vorhandene, wahrgenommene Unterschiede weiter ausgebaut und, wenn es sein musste, auch mit drastischen Klischees ausgeschmückt. ${ }^{76}$ Unbewusst wurden deutsche Vertreter vor Ort sogar zu Advokaten einer bestimmten Ethnie. ${ }^{77}$ Auf dieser Betonung und Instrumentalisierung des Ethnischen baute auch die Rekrutierung der hier untersuchten Divisionen auf. Casagrande schätzt dieses Vorgehen für die Rekrutierungsphase von „Volksdeutschen“ im Banat und Kroatien sogar als besonders wirkungsvoll ein. ${ }^{78}$ Die Gebiete, in welchen die Rekrutierungen für die vier Divisionen stattfinden sollten, gehörten wohl zu den heterogensten in Hitlers Europa. ${ }^{79}$ Im Laufe der deutschen Besetzung

72 Korb, Im Schatten des Weltkriegs, S. 28.

73 Vgl. ebd.

74 Vgl. Sundhaussen, Improvisierte Ausbeutung - der Balkan unter deutscher Okkupation, in: ten Cate, Otto, (Hgg.): Das organisierte Chaos, $59 \mathrm{f} ., 64 \mathrm{f}$.

75 Vgl. Korb, Im Schatten des Weltkriegs, S. 26.

76 Volkstumsreferent Dr. Feninger beim Bevollmächtigten des AA in Belgrad, Bericht „über die Reise in das Arnautengebiet von Kosovska Mitrovica und Novi Pazar“, 15.-26.10.1941, PAAA, R261153, Bl. 43.

77 Siehe die eben erwähnten Beispiele Pfeiffers und Feningers.

78 Vgl. Casagrande, Volksdeutsche in der Waffen-SS, S. 188.

79 Vgl. auch Korb, Im Schatten des Weltkriegs, S. 78, 82. 
Südosteuropas zwischen 1941 und 1944 sollte die ethnische Zugehörigkeit eine bisher nicht gekannte Konjunktur erleben - oft mit tödlichen Folgen. ${ }^{80}$

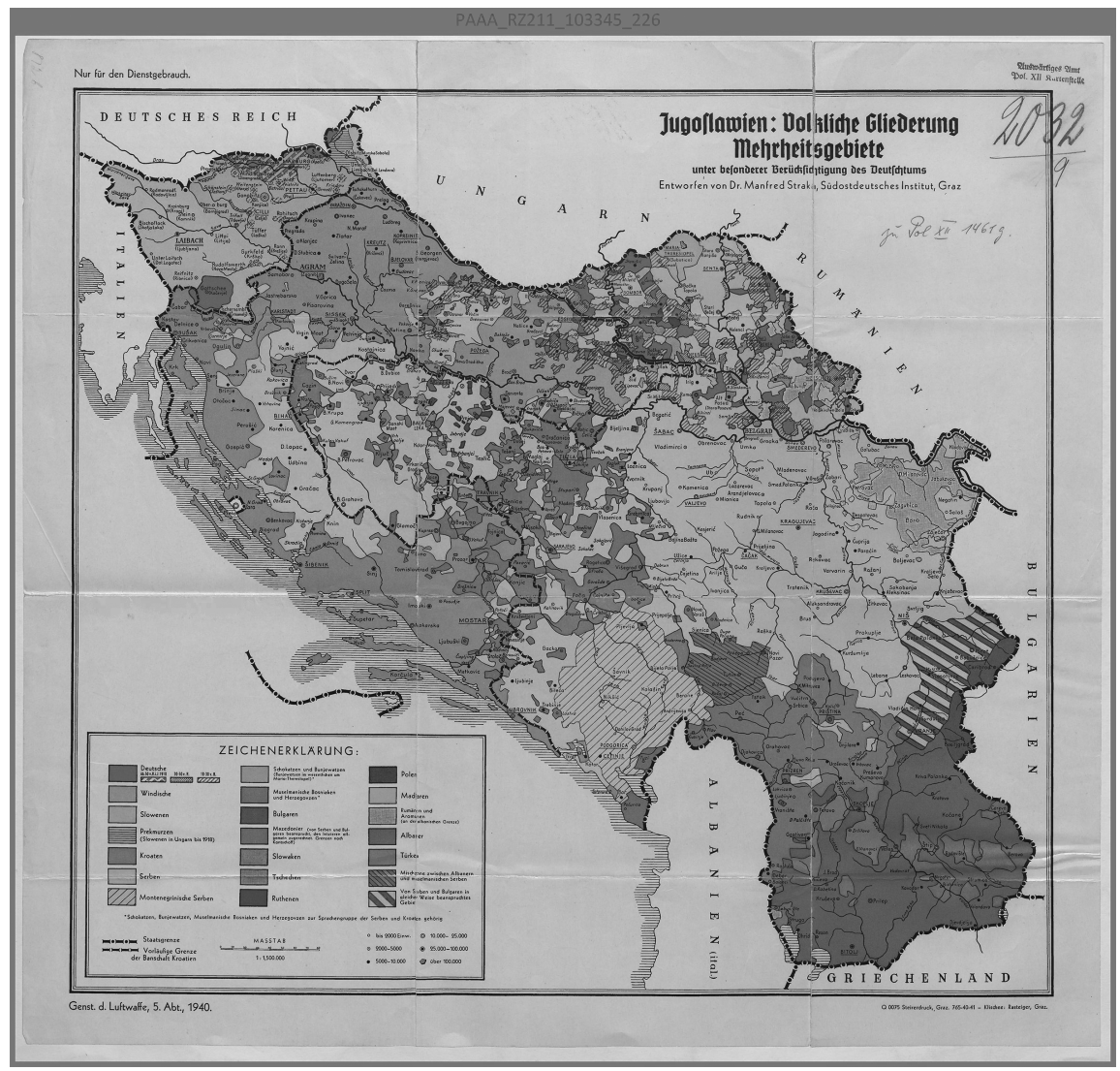

Abb. 2: „Jugoslawien: Völkische Gliederung Mehrheitsgebiete“, $1940 .^{81}$

Sechs Bürgerkriegsparteien, zwei Besatzungsarmeen und deren jeweilige Hilfstruppen ließen die Grenzgebiete zwischen Bosnien/NDH, Serbien, Montenegro und „Großalbanien“ im Chaos versinken. Dass die Kontrolle über jene interethnischen

80 Vgl. bspw. Walter Manoschek, „Serbien ist judenfrei“. Militärische Besatzungspolitik und Judenvernichtung in Serbien 1941/42, München: Oldenbourg 1993, Marko Attila Hoare, Bosnian Muslims in the Second World War oder Korb, Im Schatten des Weltkriegs.

81 „Jugoslawien: Völkische Gliederung, Mehrheitsgebiete“, Generalstab der Luftwaffe, 1940, PAAA, R103345. 
Konflikte den Besatzern aus der Hand glitt bzw. nie vollständig erreicht werden konnte, zeigt etwa folgende Tagesmeldung, welche nicht nur das Gegeneinander verschiedenster Gruppen, sondern auch die zunehmende Ohnmacht der Deutschen offenbart: „Örtliche Bandentätigkeit und anhaltender Terror beider Aufständischengruppen [Kommunisten und Četniks]. N[ord]W[estlich] Kos[ovska] Mitrovica an serbisch-albanischer Grenze Zusammenstösse zwischen Albanern und Serben. ${ }^{\text {"82 }}$ In diesen Zeitraum fiel auch die Ermordung von 360 Serben im Gefängnis von Mitrovica, die von Nedić als Akt interethnischer Gewalt von Albanern an Serben eingeordnet wurde. Eine Untersuchungskommission stellte aber fest, dass Oberleutnant Hensch die Albaner zu dieser „Sühnemaßnahme“ als Vergeltung für die Entführung eines deutschen Unteroffiziers aufgefordert hatte. Der Chef des Generalstabs des Militärbefehlshabers Südost Kurt von Geitner fürchtete um das Ansehen der Deutschen, „da diese Kommissionen und die durch sie geführten Untersuchungen im Kosovo-Gebiet bekannt geworden sind und dadurch immer wieder Tagesgespräch wurden, was geeignet ist, das deutsche Ansehen, und insbesondere das Ansehen der Polizei zu schädigen [...].“*83

\subsection{Instrumentalisierung des Islam für die Rekrutierungen der Waffen-SS}

Sowohl Hitler wie auch Himmler hegten für den Islam eine - wenn auch oberflächliche - Begeisterung. 1956 schrieb etwa Hermann Neubacher in seinen Memoiren: „Hitler hatte für den Islam sehr viel Sympathie: ,Eine Männerreligion!‘ sagte er einmal, ,wenn die Deutschen Muselmanen geworden wären, hätten sie es weiter gebracht in der Geschichte!‘ Für einen Heiden ist das ein beachtliches Zugeständnis für die Werte einer positiven Religion. Er hat aber wohl den Fatalismus des muselmanischen Kriegers im Auge gehabt. “84 Vor allem die geplante Rekrutierung von Muslimen für Waffen-SS und Wehrmacht, ließen den Islam plötzlich als der SS verwandte Religion (!) erscheinen, wie SS-Hauptsturmführer Wangemann in einer Gedächtnisaufzeichnung notierte. Er betonte weiter, „dass die politischen Interessen des Nationalsozialismus und die des Islam weitgehend

82 Chef des Generalstabs Militärbefehlshaber Südost, von Geitner and Heeresgruppe F, 18.2.1944, BArchF, RW40/85, Bl. 88.

83 Chef des Generalstabs Militärbefehlshaber Südost, Kurt von Geitner an Abwehr-Kommando 209 (H.Gr.F), 22.2.1944, BArchF, RW40/85, Bl. 111.

84 Neubacher, Sonderauftrag Südost, S. 33. Zu positiven Bemerkungen Hitlers in Bezug auf den Islam vgl. auch Werner Jochmann (Hg.), Adolf Hitler. Monologe im Führerhauptquartier 1941-1944, München: Orbis 2000, Dok. 65, 187, S. 150, 370. 
übereinstimmen [,] und dass darüber hinaus auch die weltanschaulichen Grundlagen in bestimmten Punkten auf einer Basis liegen. ${ }^{\text {(85 }}$ Die Nationalsozialisten waren aber nicht bereit, den Islam als eine Religion mit sehr unterschiedlichen Ausprägungen wahrzunehmen, vielmehr gab es für sie einfach „die islamische Welt“ bzw. „den Weltislam“. ${ }^{86}$

Denn neben der kurzfristigen Behebung von Personalengpässen verfolgten Hitler und Himmler das weiterführende Ziel, über die südosteuropäischen Divisionen die muslimischen Gemeinschaften der ganzen Welt anzusprechen. Das Versprechen auf staatliche Autonomie für rund 1,8 Millionen südosteuropäische Muslime sollte erstens dabei helfen, möglichst viele von ihnen zu rekrutieren und zweitens die „kritischen Araber“ dazu zu bewegen, den Nationalsozialisten weitere Soldaten zu schicken, wie der Abgesandte des Mufti Alim Idris bereits 1941 Legationsrat Wilhelm Melchers mitteilte. ${ }^{87}$ Auch Wangemann betonte, dass „über ihren Selbstzweck, als bosnisch-herzegowinische Division in diesem Raum hinaus, [...] diese Division den ihnen allen bekannte[n] höheren politischen Zweck [hat], die Brücke von Europa zum Weltislam zu schlagen. “88 Im Dezember 1943 bat Berger Himmler nach einer Besprechung mit dem Großmufti darum, den islamischen Geistlichen bei der Einrichtung eines „Sender[s] (Geheimsender [s]) für die gesamte mohamedanische Welt [...], um von hier aus eine intensive Propaganda nach den einzelnen mohamedanischen Gebieten hinzu betreiben“, zu unterstützen. ${ }^{89}$

Die „weltanschaulich geistige Erziehung der muselmanischen Division“ sollte aber keine Synthese zwischen Islam und Nationalsozialismus anstreben und den muslimischen Soldaten den Nationalsozialismus nicht aufzwingen. Der Schwerpunkt in der Ausbildung sollte vielmehr auf gemeinsamen Feinden, namentlich Judentum, Anglo-Amerikanismus, Kommunismus, Freimaurerei und Katholizismus (Vatikan) und „der gemeinsamen positiven Linie: a) Kämpferische Grundeinstellung, b) ethisch-sittliche Auffassung, c) völkische Erziehung “ liegen. ${ }^{90}$

85 Gedächtnisaufzeichnung der „Dienstbesprechung der Kommandeure und Imame“ Führer der Abteilung VI, SS-Hauptsturmführer Ekkehard Wangemann, 8.4.1944, BArchB, NS 19/2601, Bl. 186.

86 Vgl. Motadel, Islam, S. $3 \mathrm{f}$.

87 Vgl. Zaugg, Albanische Muslime, S. 194f. Vgl. auch Abgesandter des Mufti Alim Idris an AA, Legationsrat Wilhelm Melchers, 28.4.1941, PAAA, R103345, Bl. 174.

88 Gedächtnisaufzeichnung der „Dienstbesprechung der Kommandeure und Imame“ Führer der Abteilung VI, SS-Hauptsturmführer Wangemann, 8.4.1944, BAB, NS 19/2601, Bl. 186.

89 Berger an Himmler, betr. „Mohamedanischer Sender/Ostafrikanische mohamedanische Divisionen“, 4.12.1943, BArchB, NS 19/1896, Bl. 1.

90 Abschrift „Betr. Weltanschaulich geistige Erziehung der muselmanischen SS-Division. Bez: Mündlicher Befehl Chef SS-Hauptamt“, SS-Hauptsturmführer, Unterschrift nicht lesbar, 19.5.1943, BAB, NS 19/2601, Bl. 245. 
Als wesentliche Voraussetzung und kleinster Nenner für das Gelingen der Instrumentalisierung des Islams galt somit: ,unbedingte gegenseitige Achtung der beiden Weltanschauungen (Nationalsozialismus - Islam).“91 Auch Himmler betonte, „Sie [die Muslime] sind dem Aufruf der islamischen Führung gefolgt und sind zum Hass gegen den gemeinsamen jüdisch-englisch-bolschewistischen Feind und aus Verehrung und Treue zu dem von ihnen allen verehrten Führer Adolf Hitler gekommen.“92

Ende Februar 1944 skizzierte Himmler, wie er sich die Integration aller „waffenfähigen“ europäischen Muslime in die Waffen-SS durch eine "Mobilisierung des Islam” vorstellte: Die Rekrutierung sollte sich nicht nur auf Zivilarbeiter, sondern auch auf Zwangsarbeiter der Organisation Todt, Kriegsgefangene und weitere Kategorien in Spanien, Frankreich, Italien, Griechenland, Rumänien, Bulgarien, Albanien und Kroatien erstrecken. Um das Ziel einer möglichst umfassenden Rekrutierung europäischer Muslime zu erreichen, war Himmler auch bereit, mit dem Auswärtigen Amt und den fremden Regierungen zusammenzuarbeiten. Er stellte sich vor, dass diese Muslime schließlich in Sammellagern konzentriert würden, und der Großmufti von Jerusalem, Mohammed Amin al-Huseini danach die weitere „Werbung und politische Zielsetzung dieser Mohammedaner für den Waffendienst“ "übernehmen würde. ${ }^{93}$

91 Ebd., Hervorhebung im Original.

92 Schreiben Reichsführer SS an SS-Hauptamt, SS-Führungshauptamt, SS-Obergruppenfüher Phleps, SS-Gruppenführer Kammerhofer, SS-Obersturmbannführer Wagner, 6.8.1943, BArchB, NS19/3285.

93 Vgl. Reichsführer SS, SS-Hauptamt A I/M, Vermerk betr. „Mobilisierung des Islam“, 28.2.1944, BArchB, NS 31/42, Bl. 6. Sehr konkrete Vorstellungen hatte Berger bereits im Dezember 1943 in Bezug auf die Rekrutierung internierter muslimischer Nordafrikaner in Frankreich geäußert. Er war davon überzeugt, dass „diese Mohamedaner gern in Italien gegen Engländer und Amerikaner kämpfen“ würden. Berger an Himmler, „Mohamedanischer Sender/Ostafrikanische mohamedanische Divisionen“, 4.12.1943, BArchB, NS19/1896, Bl. 1. 


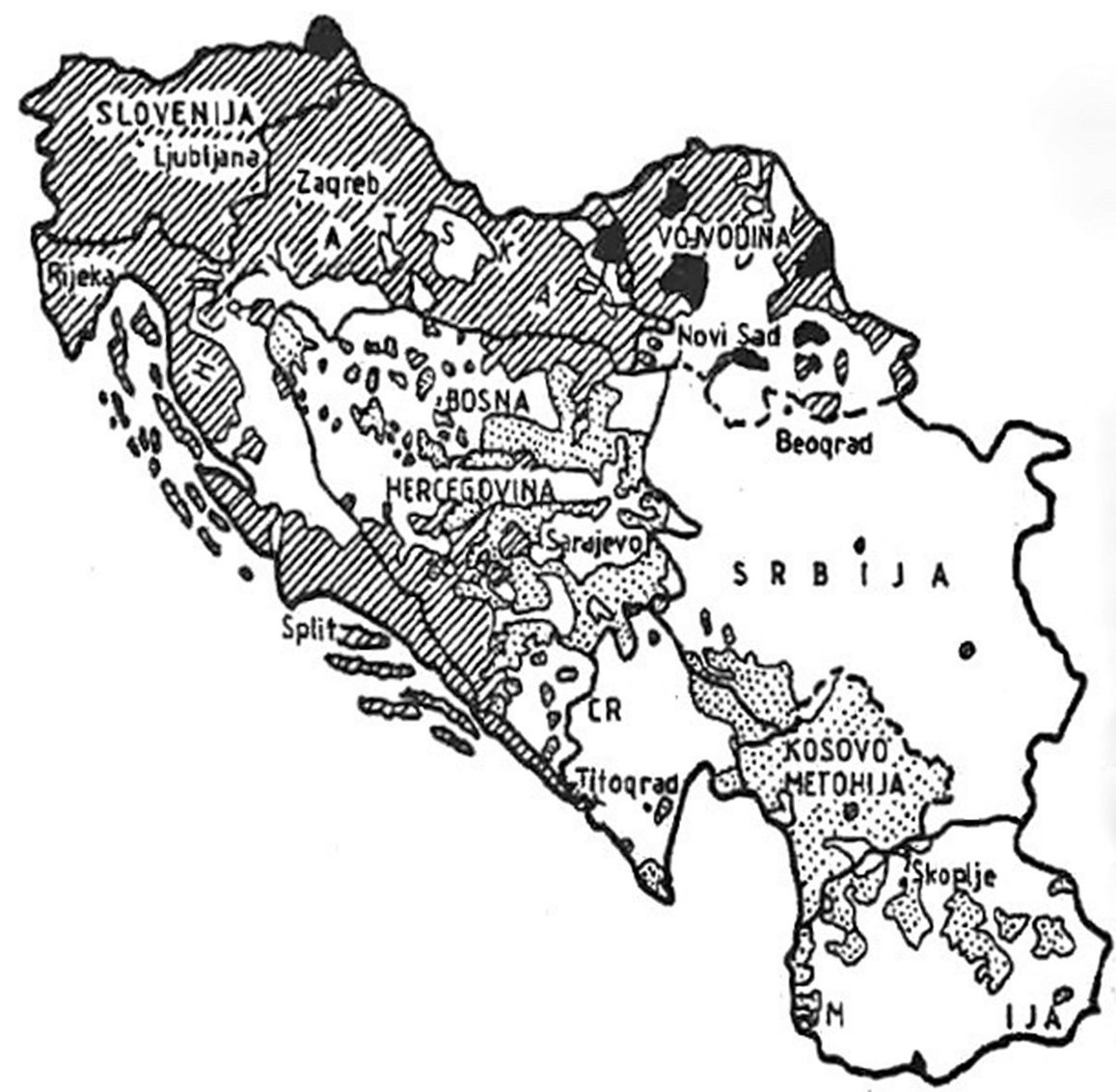

Abb. 3: Religious dominations in Yugolsavia in the 1940's: White = Orthodox, Stripes $=$ Catholics, Points $=$ Muslims, Black $=$ Protestants.$^{94}$

94 Vgl. Radmila Radić: The Serbian Orthodox Church under the Nazi Occupation and the First Years after the War 1941-1953, in: Lieve Gevers; Jan Bank, Religion under Siege II, Protestant, Orthodox and Muslim Communities in Occupied Europe (1939-1950), Leuven etc.: Petters 2007, S. 56. 